PROCEEDINGS OF THE

AMERICAN MATHEMATICAL SOCIETY

Volume 139, Number 5, May 2011, Pages 1585-1597

S 0002-9939(2010)10813-4

Article electronically published on December 15, 2010

\title{
THE SYMMETRIC OPERATION IN A FREE PRE-LIE ALGEBRA IS MAGMATIC
}

\author{
NANTEL BERGERON AND JEAN-LOUIS LODAY
}

(Communicated by Jim Haglund)

\begin{abstract}
A pre-Lie product is a binary operation whose associator is symmetric in the last two variables. As a consequence its antisymmetrization is a Lie bracket. In this paper we study the symmetrization of the pre-Lie product. We show that it does not satisfy any other universal relation than commutativity. This means that the map from the free commutative-magmatic algebra to the free pre-Lie algebra induced by the symmetrization of the pre-Lie product is injective. This result is in contrast with the associative case, where the symmetrization gives rise to the notion of a Jordan algebra. We first give a selfcontained proof. Then we give a proof which uses the properties of dendriform and duplicial algebras.
\end{abstract}

\section{INTRODUCTION}

A pre-Lie algebra is a vector space equipped with a binary operation $x * y$ whose associator is right-symmetric. Its name comes from the fact that the antisymmetrization $[x, y]:=x * y-y * x$ of this binary operation is a Lie bracket. In this paper we investigate the symmetrization $x \# y:=x * y+y * x$ of the pre-Lie product. We show that, in contrast to the bracket, it does not satisfy any universal relation (but commutativity of course). In other words the map of operads

$$
\text { ComMag } \rightarrow \text { preLie }
$$

induced by the symmetrization is injective. Here ComMag stands for the operad encoding the algebras equipped with a commutative binary operation (sometimes called commutative nonassociative algebras in the literature). We give two different proofs of this result. The first one is self-contained and relies on the combinatorics of trees. The second one uses the dendriform algebras. More precisley we prove that the map of operads

$$
M a g \rightarrow \text { Dend }
$$

is injective by using the theory of generalized bialgebras for duplicial algebras. Vladimir Dotsenko informed us that he found an alternative proof by using Groebner basis 3 .

Received by the editors May 25, 2010.

2010 Mathematics Subject Classification. Primary 16W30, 17A30, 18D50, 81R60.

Key words and phrases. Pre-Lie algebra, Jordan algebra, magmatic algebra, dendriform algebra, duplicial algebra, operad, planar tree.

(C)2010 American Mathematical Society Reverts to public domain 28 years from publication 
As a byproduct of our proof we show that the symmetrization of the binary operation generating the operad NAP induces an injective morphism

$$
\text { ComMag } \rightarrow \text { NAP. }
$$

We recall that a $N A P$-algebra is equipped with a binary operation satisfying the quadratic relation $(x y) z=(x z) y$.

\section{Prerequisite on operads}

Let Fin be the category of finite sets, and Vect be the category of vector spaces over a field $\mathbb{K}$. Let $\underline{n}=\{1, \ldots, n\}$ be the standard finite set of cardinality $n$. We will sometimes use the fact that it is equipped with a total order. Any functor Fin $\rightarrow$ Vect, $S \mapsto \mathcal{O}[S]$, gives rise to an endofunctor of Vect by the formula

$$
\mathcal{O}(V):=\bigoplus_{n \geq 1} \mathcal{O}[\underline{n}] \otimes_{\mathbb{S}_{n}} V^{\otimes n}
$$

By definition an algebraic operad is such a functor equipped with a monoid structure, i.e. transformations of functors

$$
\gamma: \mathcal{O} \circ \mathcal{O} \rightarrow \mathcal{O} \quad \text { and } \quad \iota: \operatorname{Id} \rightarrow \mathcal{O}
$$

such that $\gamma$ is associative and $\iota$ is a unit for $\gamma$. We observe that $\mathcal{O}(n):=\mathcal{O}[\underline{n}]$ is a module over the symmetric group $\mathbb{S}_{n}$.

We recall that a type of algebras gives rise to an algebraic operad by taking the free algebra functor (cf. for instance [11, 8]).

\section{Pre-Lie Algebra}

A pre-Lie algebra is a vector space $L$ together with a binary operation $*$ satisfying the relation

$$
(x * y) * z-x *(y * z)=(x * z) * y-x *(z * y), \quad \forall x, y, z \in L .
$$

The operad $\mathcal{P}=$ preLie has been described in terms of trees by Chapoton and Livernet in [2] as follows. Given a finite set $S$ of cardinality $n$, let $\mathcal{P}[S]$ be the vector space spanned by the labelled rooted trees on $n$ vertices with distinct labels chosen in $S$. For example the space $\mathcal{P}[\{a, b, c\}]$ is the linear span of the following trees:

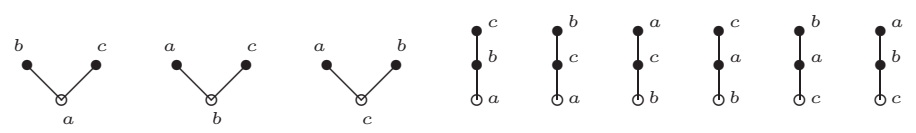

The pre-Lie product is obtained by the following process. Given two disjoint sets $I, J$ and two labelled trees $T \in \mathcal{P}[I]$ and $Y \in \mathcal{P}[J]$ one defines

$$
T * Y:=\sum_{t \in V \operatorname{ert}(T)}
$$

where the sum is over all possible ways of grafting the root of the tree $Y$ on a vertex $t$ of $T$ by an edge. The root of the result is the root of $T$. For example

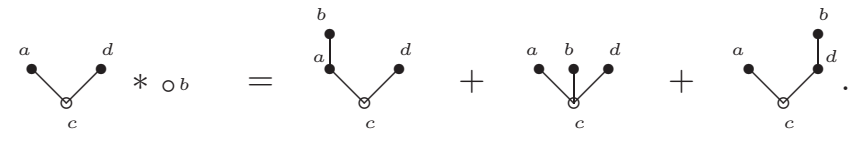


Any pre-Lie algebra $L$ gives rise to a Lie algebra whose bracket is defined by the antisymmetric product

$$
[x, y]:=x * y-y * x .
$$

This gives a morphism of operads

$$
\text { Lie } \longrightarrow \text { preLie. }
$$

This morphism is injective and has been well studied in the literature; cf. for instance [2] and [9.

In this paper we consider the symmetric product

$$
x \# y:=x * y+y * x .
$$

This new binary operation is related to the commutative-magmatic operad ComMag, where a ComMag-algebra is a vector space $A$ together with a binary operation $\cdot$ satisfying the relation

$$
x \cdot y=y \cdot x, \quad \forall x, y \in A .
$$

The symmetric product \# in preLie gives a morphism of operads

$$
\Phi: \text { ComMag } \longrightarrow \text { preLie. }
$$

This has been much less studied, and our main theorem is to show that this morphism is injective. Let us recall that $\operatorname{ComMag}(n)$ is of dimension $(2 n-3) ! !=$ $1 \times 3 \times \cdots \times(2 n-3)$ and that preLie $(n)$ is of dimension $n^{n-1}$.

An element in ComMag $[S]$ is a product $x \cdot y$ where $x \in \operatorname{ComMag}[I], y \in$ ComMag $[J]$ for some decomposition $S=I \cup J$ (disjoint union). Let us suppose that $S$ is equipped with a total order. Thanks to the commutativity of the product we can assume that the label $\max (S)$ is in $J$. In fact, there is a unique way to write an element in $C o m M a g[S]$ such that $\max (S)$ is on the right side and the two factors $x$ and $y$ also satisfy this property. We call it the normalized writing. For instance the elements of $\operatorname{ComMag}(3)$ are written

$$
(1 \cdot 2) \cdot 3, \quad 1 \cdot(2 \cdot 3), \quad 2 \cdot(1 \cdot 3) .
$$

We can now define $\Phi: C o m M a g \rightarrow \mathcal{P}$ explicitly. If $S=\{a\}$ is of cardinality one, then $\Phi(a)=a$. If $|S|>1$, then any $Y \in \operatorname{ComMag}[S]$ decomposes uniquely as $Y=V \cdot W$. We let $\Phi(Y):=\Phi(V) \# \Phi(W)$ and extend linearly to ComMag $[S]$.

\section{Symmetrizing the PRe-Lie PRoduct}

The main result of this paper is the following:

3.1. Theorem. The morphim of operads $\Phi:$ ComMag $\rightarrow$ preLie induced by the symmetric product $x \# y$ is injective.

Proof. Plan of the proof. We introduce the auxiliary operation $x \tilde{*} y$ on the labelled trees as follows. For any labelled trees $T$ and $Y$ (labelled on disjoint finite sets), $T \approx \widetilde{*} Y$ is the unique tree obtained by connecting the root of $Y$ to the root of $T$. The root of $T \tilde{*} Y$ is the former root of $T$ :

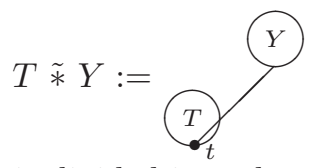

The proof of the theorem is divided into three steps. 
Step 1. We show that it is sufficient to prove injectivity for the symmetrization of the operation $\tilde{*}$ (in place of $*$ ).

Step 2. We identify a subset of $\mathcal{P}[S]$ which is in bijection with $\operatorname{ComMag}[S]$ and we show that $\tilde{*}$ realizes this bijection.

Step 3 . We show that the symmetrization of the operation $\tilde{*}$ induces an injective map $C o m M a g[S] \rightarrow \mathcal{P}[S]$.

Step 1. We need to show that the linear maps $\Phi_{S}: \operatorname{ComMag}[S] \rightarrow \mathcal{P}[S]$ are injective for all finite sets $S$. We first introduce a filtration on $\mathcal{P}[S]$. For a tree $T \in \mathcal{P}[S]$, we say that $T$ is of degree $d$ if the number of subtrees connected to the root of $T$ is $d$. We write $\operatorname{deg}(T)=d$. This induces a filtration of $\mathcal{P}[S]$. The pre-Lie product with respect to this filtration is

$$
T * Y=T \tilde{*} Y+\text { lower degree terms, }
$$

where $T \tilde{*} Y$ is the unique tree obtained by connecting the root of $Y$ to the root of $T$. So, for the purpose of this proof, we may use the product $\tilde{*}$ instead of $*$.

Step 2. We define a map $\Psi: \operatorname{ComMag}[S] \rightarrow \mathcal{P}[S]$, we identify its image, and finally we prove that it is injective.

Let $S$ be a totally ordered finite set. Let $t=x \cdot y$ be an element in $\operatorname{ComMag}[S]$ whose writing has been normalized; that is, $\max (S)$ is a label of $y$. We define inductively

$$
\Psi(t):=\Psi(x) \tilde{*} \Psi(y),
$$

starting with $\Psi(1)=\circ_{1}$. So, we get

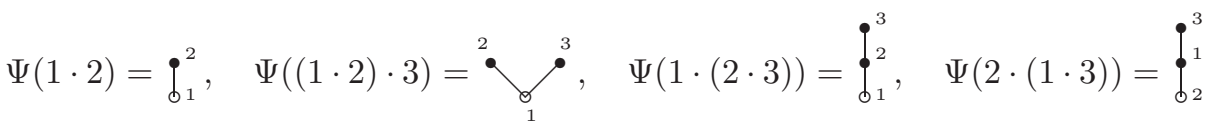

Any tree $T \in \mathcal{P}[S]$ is uniquely determined by its root $r$ and a set of subtrees $\left\{T_{1}, T_{2}, \ldots, T_{\ell}\right\}$ attached to the root. We denote this decomposition as $T=$ $\left(r,\left\{T_{1}, T_{2}, \ldots, T_{\ell}\right\}\right)$. For $i \in\{1,2, \ldots, \ell\}$ let $b_{i}=\max \left(T_{i}\right)$. We can assume that $b_{1}<b_{2}<\cdots<b_{\ell}$. For a given set $S$, we denote by $A[S]$ the linear span of the trees in the image $\Psi[S]$ and by $B[S]$ the linear span of the trees of $\mathcal{P}[S]$ not in $A[S]$. We have that $\mathcal{P}[S]=A[S] \oplus B[S]$. For any tree $T \in \mathcal{P}[S]$, either the root is labelled with $\max (T)$ or there is a unique factorization

$$
T=X_{1} \tilde{*} X_{2},
$$

where $\max (T)=b_{\ell}$ is a label in $X_{2}=T_{\ell}$. For the factorization in (10) to exist in $A[S]$ we must have that $r$ is not $\max (T)$. That is, $r<b_{\ell}$. We remark that, recursively, $X_{1}$ will be factorizable if $r>b_{\ell-1}$. As we repeat this we see that $T \in B[S]$ if $r>b_{1}$. In particular, we get a characterization of the $T \in A[S]$ as follows.

Claim 1. The image $\Psi(x \cdot y)$ is a tree $T=\left(r,\left\{T_{1}, T_{2}, \ldots, T_{\ell}\right\}\right)$, which is characterized by the following properties:

(a) $T_{i} \in A\left[S_{i}\right]$ for all $i \in\{1,2, \ldots, \ell\}$;

(b) $r<b_{1}$.

Proof of Claim 1. The tree $T_{i}$ either comes from $\Psi(x)$ or is $\Psi(y)$. In both cases it is in $A\left[S_{i}\right]$ by induction. The index $b_{i}$ either comes from $\Psi(x)$ or is $\max \Psi(y)=$ $\max (y)$. In both cases we have $r<b_{i}$ as expected.

Claim 2. $\Psi$ is a bijection onto its image $A[S]$. 
Proof of Claim 2. Starting with $T \in \operatorname{Im} \Psi$, by claim 1 we have $T=T_{1} \tilde{*} T_{2}$, where $\max (T) \in T_{2}$ and $T_{1}=\Psi\left(x_{1}\right), T_{2}=\Psi\left(x_{2}\right)$. So we have $T=\Psi\left(x_{1} \cdot x_{2}\right)$. So we have constructed $\Psi^{-1}$. This shows that $\Psi$ is an isomorphism from ComMag $[S]$ to $A[S]$.

Step 3. For $T \in A[S]$ and its factorisation $T=X_{1} \tilde{*} X_{2}$, for which $\max (T)=$ $\max \left(T_{2}\right)$, we define $d(T)=\left|X_{2}\right|$, the size of $X_{2}$ (the number of vertices).

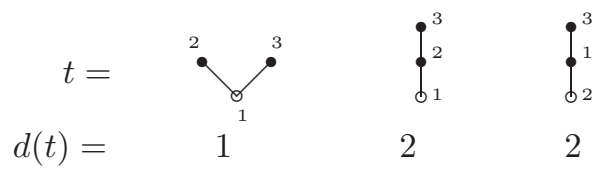

We can now turn to the map $\widetilde{\Phi}: \operatorname{ComMag}[S] \rightarrow \mathcal{P}[S]$ given by

$$
\widetilde{\Phi}(x \cdot y)=\Phi(x) \widetilde{\#} \Phi(y):=\Phi(x) \tilde{*} \Phi(y)+\Phi(y) \tilde{*} \Phi(x) .
$$

Our purpose is to show that it is injective. Given a tree $Y \in \operatorname{ComMag}[S]$ the tree $\Psi(Y) \in \mathcal{P}[S]$ is clearly in the support of $\widetilde{\Phi}(Y)$. We want to show that the image of $\widetilde{\Phi}[S]$ is linearly isomorphic to $A[S]$. This would show the injectivity of our map. The principle of the proof is to show that in $\widetilde{\Phi}(T)$, several elements of $A[S]$ of various $d$ values may appear, but there is only one element of $A[S]$ of maximal $d$ value. For instance $\widetilde{\Phi}(1 \cdot(2 \cdot 3))$ contains only one element $T_{1} \in A[S]$, where $d\left(T_{1}\right)=2, \widetilde{\Phi}((1 \cdot 2) \cdot 3)$ contains only one element $T_{2} \in A[S]$, where $d\left(T_{2}\right)=1$, while $\widetilde{\Phi}(2 \cdot(1 \cdot 3))$ contains two elements $T_{2}, T_{3} \in A[S]$, where $d\left(T_{3}\right)=2$ :

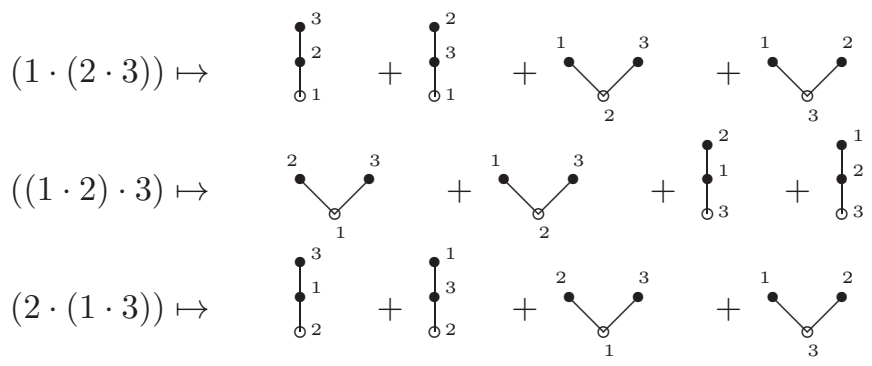

We proceed by induction on $|S|$. If $|S|=1$, then $\widetilde{\Phi}$ is a linear isomorphism and the result is clear. Assume $|S|>1$. Our induction hypothesis is that the image of $\widetilde{\Phi}\left[S^{\prime}\right]$ is isomorphic to $A\left[S^{\prime}\right]$ for all $\left|S^{\prime}\right|<|S|$. More precisely, we assume that the image of $\widetilde{\Phi}\left[S^{\prime}\right]$ is exactly $A\left[S^{\prime}\right]$ modulo terms in $B\left[S^{\prime}\right]$. This is certainly true for $|S|=1$.

To obtain this, we order the trees of $A[S]$ (a basis) as follows. Let $T, T^{\prime}$ be trees in $A[S]$. We say that $T<T^{\prime}$ whenever $d(T)>d\left(T^{\prime}\right)$. When $|S|>1$ we have that

$$
\operatorname{ComMag}[S]=\bigoplus_{\substack{S=S_{1}+S_{2} \\ \min \left(S_{1}\right)<\min \left(S_{2}\right)}} \operatorname{ComMag}\left[S_{1}\right] \cdot \operatorname{ComMag}\left[S_{2}\right],
$$

where $S=S_{1}+S_{2}$ denotes a set partition of $S$ into two nonempty disjoint parts. This implies that

$$
\widetilde{\Phi}[S]=\bigoplus_{\substack{S=S_{1}+S_{2} \\ \min \left(S_{1}\right)<\min \left(S_{2}\right)}} \widetilde{\Phi}\left[S_{1}\right] \tilde{\#} \widetilde{\Phi}\left[S_{1}\right],
$$

where $V \widetilde{\#} W=V \tilde{*} W+V \tilde{*} W$. For $i=1$ or 2 , by the induction hypothesis, we may assume that a basis of the image of $\widetilde{\Phi}\left[S_{i}\right]$ is of the form $\left\{X_{i}+W_{i}: X_{i} \in A\left[S_{i}\right]\right\}$, 
where the $X_{i}$ 's are single trees and $W_{i} \in B\left[S_{i}\right]$. Let $T$ be any tree in $A[S]$. Using the decomposition in (11), we have that there are unique $S=S_{1}+S_{2}$ and trees $X_{1} \in A\left[S_{1}\right]$ and $X_{2} \in A\left[S_{2}\right]$ such that $T=X_{1} \tilde{*} X_{2}$. By the induction hypothesis, there are basis elements $X_{1}+W_{1}$ in the image of $\widetilde{\Phi}\left[S_{1}\right]$ and $X_{2}+W_{2}$ in the image of $\widetilde{\Phi}\left[S_{2}\right]$. By construction we have that

$$
\left(X_{1}+W_{1}\right) \tilde{\#}\left(X_{2}+W_{2}\right)=X_{1} \tilde{*} X_{2}+X_{2} \tilde{*} X_{1}+\cdots .
$$

We study this equation. In the following, let $b=\max (T)$. Remark that $b$ is necessarily a label in $X_{2}$.

Let $T^{\prime}=X_{2} \tilde{*} X_{1}$. If $T^{\prime} \in A[S]$, then $b$ must be in a proper subtree $V_{2}$ of $T^{\prime}$ and the decomposition as in (11) for $T^{\prime}$ is $T^{\prime}=V_{1} \tilde{*} V_{2}$. We remark that $V_{2}$ must be a proper subtree of $X_{2}$ (since the root of $T^{\prime}$ is the root of $X_{2}$ ); hence $\left|X_{2}\right|>\left|V_{2}\right|$. This implies that $T<T^{\prime}$. It may happen that $T^{\prime} \in B[S]$, but this does not cause any problem. We need to inspect the other terms in (2). Let $Z_{i}$ be a tree in the support of $W_{i}$, in particular $Z_{i} \in B\left[S_{i}\right]$. For any tree $Y$, the tree $Z_{i}$ will eventually be a factor as in (11) for the tree $Y \tilde{*} Z_{i}$ (this may take more than one recursive step). This shows that $\left(X_{1}+W_{1}\right) \tilde{*} W_{2}$ and $\left(X_{2}+W_{2}\right) \tilde{*} W_{1}$ are both in $B[S]$. For elements of the form $Z_{i} \tilde{*} Y$ we have to be more careful. For $Z=Z_{1} \tilde{*} X_{2}$, we have that $\max (Z)=b$ is in $X_{2}$. This implies that $Z=Z_{1} \tilde{*} X_{2}$ is the unique decomposition of $Z$ as in (11), so $Z \notin A[S]$. This shows that $W_{1} \tilde{*} X_{2} \in B[S]$. Finally, for $Z=Z_{2} \tilde{*} X_{1}$, if $Z \in A[S]$, then $b$ must be in a proper subtree $V_{2}$ of $Z$ and the decomposition as in (10) for $Z$ is $Z=V_{1} \tilde{*} V_{2}$. We remark that $V_{2}$ must be a proper subtree of $Z_{2}$; hence $\left|X_{2}\right|=\left|Z_{2}\right|>\left|V_{2}\right|$. This implies that $T<Z$. For all $T \in A[S]$, we have shown that there is an element in the image of $\widetilde{\Phi}[S]$ given by (2) which takes the form

$$
\left(X_{1}+W_{1}\right) \widetilde{\#}\left(X_{2}+W_{2}\right)=T+\sum_{\substack{T^{\prime} \in A[S] \\ T<T^{\prime}}} c_{T^{\prime}} T^{\prime}+W
$$

where $W \in B[S]$.

We have produced elements in the image of $\widetilde{\Phi}[S]$ that are linearly independent (different leading terms), one for each tree $T \in A[S]$. Since $\operatorname{dim}(A[S])=$ $\operatorname{dim}(\operatorname{ComMag}[S])$ we have that $\widetilde{\Phi}[S]$ is injective.

\section{Comparison with associative, Jordan and NAP-algebras}

4.1. NAP-algebras. The operation $\tilde{*}$ used as an auxiliary operation in the proof of the main theorem satisfies the following relation:

$$
(x \tilde{*} y) \tilde{*} z=(x \tilde{*} z) \tilde{*} y
$$

for any labelled trees $x, y, z$. Algebras with one binary operation satisfying this identity have already occurred in the literature in the work of M. Livernet [4, and were called NAP-algebras (for NonAssociative Perm). She showed that the labelled trees do describe the associated operad and that the product is precisely given by the operation $\tilde{*}$ on trees described in the proof of Theorem 3.1. Therefore, as a by-product of the proof of the main theorem we obtain the following:

4.2. Theorem. The symmetrization of the operation $\tilde{*}$ induces an injection of operads

$$
\text { ComMag } \rightarrow \text { NAP. }
$$


4.3. Jordan algebras. Let $x \cdot y:=x y+y x$, where $x y$ is an associative operation. It is easy to check that the 3 operations $(x \cdot y) \cdot z,(x \cdot z) \cdot y,(y \cdot z) \cdot x$ are linearly independent in the free associative algebra. However the 15 operations in arity 4 are not linearly independent: they satisfy the formula

$(x \cdot y) \cdot(t \cdot z)+(x \cdot z) \cdot(t \cdot y)+(y \cdot z) \cdot(t \cdot x)=((x \cdot y) \cdot t) \cdot z+((x \cdot z) \cdot t) \cdot y+((y \cdot z) \cdot t) \cdot x$.

Since this formula is invariant under any permutation of the three variables $x, y, z$ it is equivalent, when 3 is invertible, to a formula in two variables only. It leads to the definition of a Jordan algebra, which is a vector space equipped with a symmetric binary operation $a \cdot b$ satisfying the relation

$$
\left(a^{2}\right) \cdot(b \cdot a)=\left(\left(a^{\cdot 2}\right) \cdot b\right) \cdot a .
$$

Let us denote by Jord the operad governing Jordan algebras. Since any symmetrized associative product satisfies the Jordan relation there is a morphism of operads

$$
\text { Jord } \rightarrow \text { As . }
$$

Contrary to the Lie-As case and the ComMag-preLie case, this morphism is not injective. In other words there are relations satisfied by the symmetrized associative product which are not consequences of the Jordan relation. The first examples appear in arity 8: the Glennie relations; see p. 79 of [13].

\section{Conjecture on the factorization of preLie}

5.1. On the injectivity of Lie $\rightarrow A s$. There are several ways of proving the injectivity of the morphism of operads Lie $\rightarrow$ As. One of them is to take advantage of the existence of the notion of "cocommutative bialgebra". Indeed, the PoincaréBirkhoff-Witt theorem implies that there is an isomorphism of endofunctors of Vect:

$$
\text { As }=\text { Com } \circ \text { Lie. }
$$

Therefore it is natural to look for a similar statement when replacing Lie-As by ComMag-preLie.

5.2. Conjecture: splitting of preLie. There exists an $\mathbb{S}$-module $\mathcal{X}$ such that

$$
\text { preLie }=\mathcal{X} \circ \text { ComMag. }
$$

From this formula the dimension of the spaces $\mathcal{X}(n)$ would be

$$
1,1,3,16,120,1146,13258, \ldots
$$

5.3. Black and red trees. Since the conjecture is only about the structure of the $\mathbb{S}$-module of preLie and since $N A P$ has the same underlying $\mathbb{S}$-module we could as well put $N A P$ in the statement. For the rest of the section we denote by $\mathcal{P}$ the underlying $\mathbb{S}$-module of both preLie and $N A P$.

Here are some arguments in favor of the conjecture. In this section we construct a linear isomorphism

$$
\mathcal{P}[S] \longrightarrow \bigoplus_{\phi \vdash S} \mathcal{X}[\phi] \times \prod_{R \in \phi} \operatorname{ComMag}[R],
$$


where $\phi \vdash S$ denotes a set partition of $S$. Unfortunately, the family of spaces $\mathcal{X}$ that we construct is not defining an $\mathbb{S}$-module. We believe that our construction can be modified to realize this, but we have not yet found the right way to do so.

In Step 2 of Section 3 we constructed an injection $\Psi: C o m M a g \rightarrow \mathcal{P}$. We say that a tree of $\mathcal{P}$ that is in the image of $\Psi$ is a tree of type $A$. In Claim 1 of Step 2 we have a characterization of the tree of type $A$. To obtain the linear isomorphism in (4), we decompose (uniquely) any tree of $\mathcal{P}$ into special trees (of type $\mathcal{X}$ ) labelled with trees of type $A$.

To get this decomposition, we work recursively from the leaves down to the root. We will mark some edges red (in the electronic version, double lines in the print version) and others will remain black. The black connected components will represent the trees of type $A$ and the double red edges will connect them to form a tree. We also have that a double red edge is always between the root of a black tree to the maximum in the black tree below connected to it by that double red edge.

If $T$ is a single leaf, then there are no edges to color. Now assume we have $T=\left(r,\left\{T_{1}, T_{2}, \ldots, T_{\ell}\right\}\right)$ and all the trees $T_{i}$ for $i \in\{1,2, \ldots, \ell\}$ have been colored. Let $T_{i}^{\prime}$ be the black connected component at the root of $T_{i}$. Let $b_{i}^{\prime}=\max \left(T_{i}^{\prime}\right)$ and assume $b_{1}^{\prime}<b_{2}^{\prime}<\cdots<b_{\ell}^{\prime}$. We assume (by construction up to that point) that in $T_{i}$, all the double red edges connected to $T_{i}^{\prime}$ are connected to $b_{i}^{\prime}$. If $r>b_{1}^{\prime}$ or if $r<b_{1}^{\prime}$ and $T_{i}^{\prime} \neq T_{i}$ for $1 \leq i<\ell$, then we mark double red all edges out of the root $r$; otherwise, we mark them black. We proceed to the next step. This will mark the edges of $T$ double red or black. If we cut all marked edges, thanks to Claim 1 we get a forest of trees of type $A$. They are connected by marked edges, and this structure forms a tree. This decomposition is unique. Remark that the condition ensures that the bicolored tree $T$ that we obtain is black at $r$ only if all double red edges in the black connected component of $r$ are connected to the maximum vertex of that component. Otherwise, the tree is double red at $r$.

The tree below show how the special double red marking works. Let

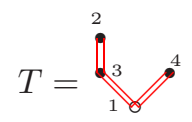

At the root 1 of $T$, we have that $1<3$, but the tree containing 3 is not all black, so we are marking the edges red (in the electronic version, double lines in the print version). If we look at the tree

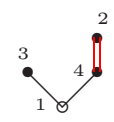

at 1 , then we see that the subtree 3 is all black and the only double red edges are connected to 4 , the maximum, so the edges out of 1 are black. 
For example we give a list of the decomposition for all trees of $\mathcal{P}[\{1,2,3,4\}]$ :

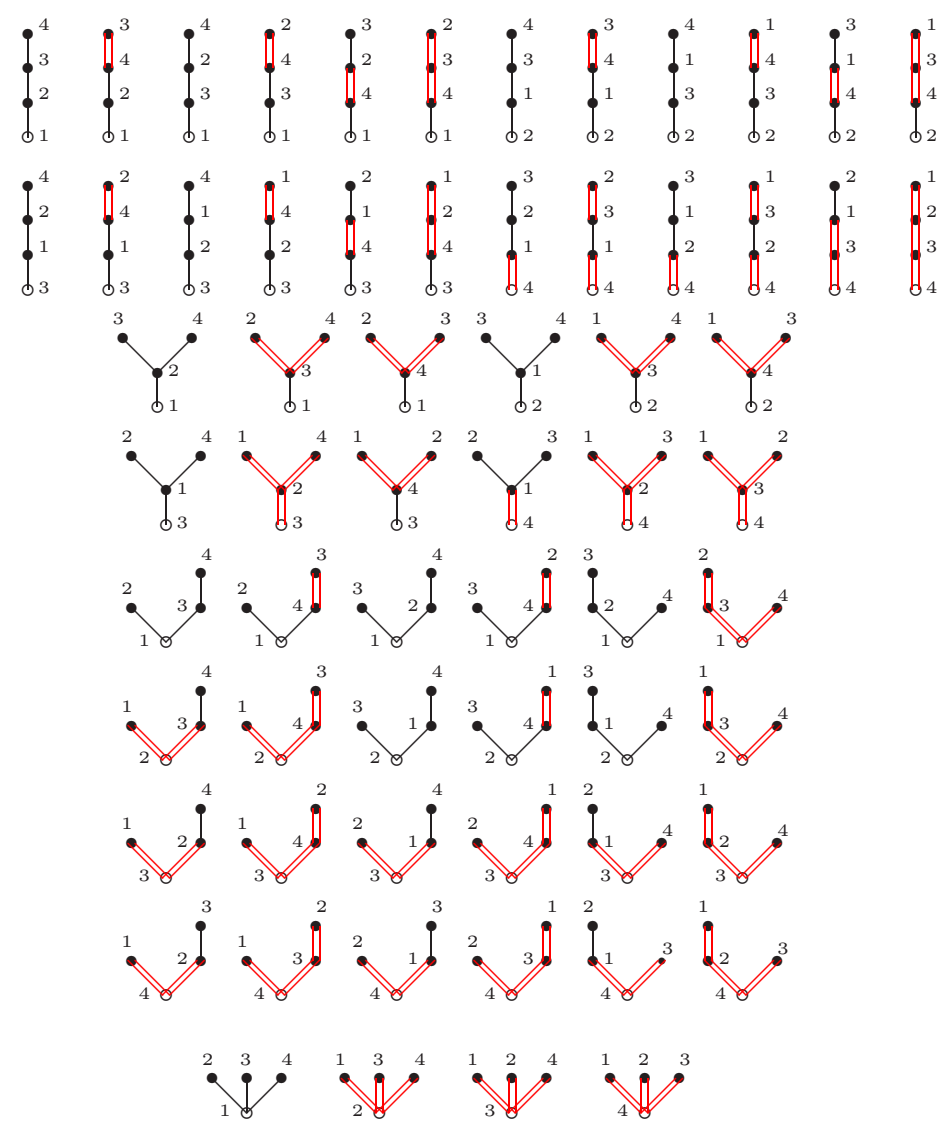

This decomposes each tree of $\mathcal{P}[S]$ in a unique way. A more careful look at this decomposition gives us the following claim.

Claim 3. The subset of trees $\mathcal{X}[S] \subseteq \mathcal{P}[S]$ that are all double red are characterized as follows. If $|S|=1$, then $\mathcal{X}[S]=\mathcal{P}[S]$. If $|S|>1$, then let $T=$ $\left(r,\left\{T_{1}, T_{2}, \ldots, T_{\ell}\right\}\right)$, let $r_{i}$ be the root of $T_{i}$ and assume $r_{1}<r_{2}<\cdots<r_{\ell}$. We have $T \in \mathcal{X}[S]$ if and only if

(a) $T_{i} \in \mathcal{X}\left[S_{i}\right]$ for all $i \in\{1,2, \ldots, \ell\}$ and

(b) $r>r_{1}$ or $\left(r<r_{1}\right.$ and $\ell>1$ and at least one $T_{i}$ for $i<\ell$ has size strictly greater than 1).

This gives us all the needed ingredients for the isomorphism in (4). For any $T \in \mathcal{P}[S]$ the unique black and double red tree we obtain gives us a set partition $\phi \vdash S$ induced by the connected black components. On each black component we have a tree of type $A$. This decomposition also induces a double red tree on $\phi$ where each part $R \in \phi$ is identified with its maximal element. This gives us a bijection (of basis) between the trees in $\mathcal{P}[S]$ and $\bigoplus_{\phi \vdash S} \mathcal{X}[\phi] \times \prod_{R \in \phi}$ ComMag $[R]$. The inverse map is simply: connect the trees of type $A$ (identified to trees in $\operatorname{ComMag}[R]$ ) according to $\mathcal{X}[\phi]$ from the root to the maximum of the tree it attaches to. 
For example for

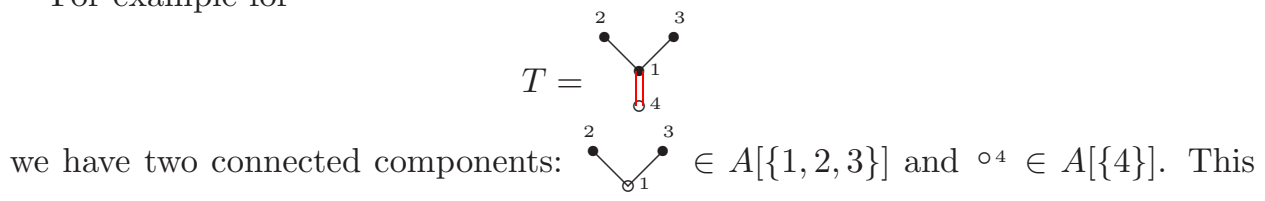

gives us the following set partition $\{\{1,2,3\},\{4\}\}$. We labelled them 3 and 4 respectively. The double red edges induce the following tree $\mathfrak{b}_{4}^{3} \in \mathcal{X}[\{3,4\}]$. It is clear how to reconstruct $T$ from this data knowing that we have to connect the root of ${ }_{1}^{3}$ to the maximal element in the tree $b_{4}^{3}$.

5.4. Bimodule structure. Comment from Vladimir Dotsenko: the behavior of the double red trees suggests that the $\mathbb{S}$-module $\mathcal{X}$ itself can be decomposed as $\mathcal{X}=$ ComMag $\circ \mathcal{Z}$ for some $\mathbb{S}$-module $\mathcal{Z}$. Computation of the power series agrees with this statement. That would make preLie into a free bimodule over ComMag.

5.5. Comment on the splitting of preLie and NAP. Since preLie (resp. $N A P)$ and ComMag are operads it is natural to ask oneself if there could be an operad structure on $\mathcal{X}$ which would be compatible with the other operad structures under the isomorphism of $\mathbb{S}$-modules. Analogously, it is natural to ask for "good

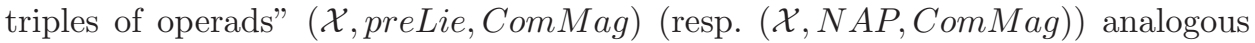
to the good triple (Com, As, Lie); cf. [7.

\section{Comparison With THE OPERAD OF DENDRIFORM ALGEBRAS}

We give another proof of Theorem 3.1 based on the comparison between the operad preLie and the operads Dend and Dup. First, we recall results about dendriform algebras and their parametrized version. Second, we recall results of [7, Chapter 5, about the operad Dup encoding duplicial algebras. Finally we give an alternative proof to the injectivity of the map ComMag $\rightarrow$ preLie .

6.1. Parametrized dendriform algebras. Let $\lambda \in \mathbb{K}$ be a parameter. We define a parametrized dendriform algebra as a vector space $A$ equipped with two binary operations

$$
\prec: A \otimes A \rightarrow A \quad \text { and } \quad \succ: A \otimes A \rightarrow A
$$

called the left operation and the right operation, respectively, satisfying the following three relations:

$$
\left\{\begin{array}{rl}
(x \prec y) \prec z & =x \prec(y \prec z)+\lambda x \prec(y \succ z), \\
(x \succ y) \prec z & =x \succ(y \prec z), \\
\lambda(x \prec y) \succ z+(x \succ y) \succ z & x \succ(y \succ z) .
\end{array}\right.
$$

For $\lambda=1$ it is the notion of a dendriform algebra introduced in [5, 6] and for $\lambda=0$ it is the notion of a duplicial algebra introduced in [7, 12. The relevant operads are denoted by Dend and Dup respectively.

We introduce the operation $\{x, y\}$ given by

$$
\{x, y\}:=x \prec y-y \succ x,
$$


and the operation $x \square y$ given by

$$
x \square y:=x \prec y-x \succ y .
$$

In the dendriform case, $\{x, y\}$ is known to be a pre-Lie product.

6.2. Lemma. The following square of categories of algebras, resp. of operads (cf. [8]), where the upper horizontal arrow is induced by $\{x, y\}$, the left vertical arrow is induced by $x \square y$ and the two other arrows are symmetrization,
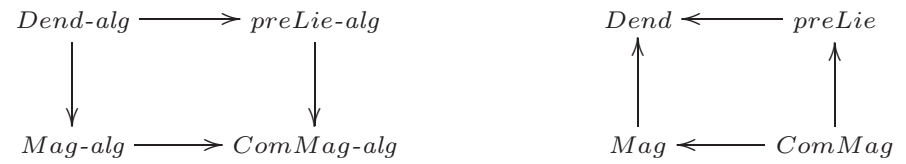

is commutative.

Proof. The commutativity of the diagram follows from the equalities

$$
\begin{aligned}
x \square y+y \square x=(x \prec y & -x \succ y)+(y \prec x-y \succ x) \\
& =(x \prec y-y \succ x)+(y \prec x-x \succ y)=\{x, y\}+\{y, x\} .
\end{aligned}
$$

6.3. On duplicial algebras. As mentioned above a duplicial algebra is defined by two binary operations $x \prec y$ and $x \succ y$ satisfying three relations:

$$
\left\{\begin{aligned}
(x \prec y) \prec z= & x \prec(y \prec z), \\
(x \succ y) \prec z= & x \succ(y \prec z), \\
(x \succ y) \succ z & =x \succ(y \succ z) .
\end{aligned}\right.
$$

There is a notion of generalized bialgebra which is duplicial as an algebra and coassociative as a coalgebra. The primitive part is an algebra encoded by the operad Mag. The generating operation of the magmatic operad is given by $x \square y=$ $x \prec y-x \succ y$ in terms of the duplicial operations. Since the "triple of operads" (Com, Dup, Mag) is good (Corollary 5.2.6 of [7]), there is an isomorphism Dup $\cong$ $A s \circ M a g$, and therefore the map Mag $\rightarrow$ Dup is injective.

6.4. Lemma. The map of ns operads Mag $\rightarrow$ Dend induced by the operation $x \square y:=x \prec y-x \succ y$ is injective.

Proof. The free $\lambda$-Dend-algebra on a generator $x$, that is, $\lambda$ - $\operatorname{Dend}(x) \cong$ $\bigoplus_{n \geq 1} \lambda$-Dend $d_{n}$, is isomorphic to $\bigoplus_{n \geq 1} \mathbb{K}\left[P B T_{n+1}\right]$. The proof is the same as the proof for $\lambda=1$ given in [6]. The inverse of this isomorphism, which we denote by $\varphi$, is obtained as follows:

$$
\varphi(Y)=x, \quad \varphi(r \vee s)=\varphi(r) \succ x \prec \varphi(s),
$$

where $r$ and $s$ are planar binary trees.

Letting $\lambda=1$, resp. $\lambda=0$, we get, for any $n$, isomorphisms of vector spaces

$$
\operatorname{Dend}_{n} \cong \mathbb{K}\left[P B T_{n+1}\right] \cong \operatorname{Dup}_{n} .
$$


We consider, in $\operatorname{Dend}(x)$ and $\operatorname{Dup}(x)$, respectively, the subspace generated by $x$ under the operation $\square$. It give rise to the following commutative diagram (of graded vector spaces):

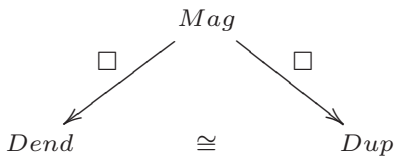

Since the map $M a g \rightarrow$ Dup is injective, so is the map $M a g \rightarrow$ Dend.

6.5. Alternative proof of Theorem 3.1. Since the map of operads ComMag $\rightarrow$ Mag is obviously injective, Lemma 6.4 implies that the composite ComMag $\rightarrow$ $\mathrm{Mag} \rightarrow$ Dend is injective. Since this is also the composite ComMag $\rightarrow$ Pre-Lie $\rightarrow$ Dend by Lemma 6.2 the map ComMag $\rightarrow$ Pre-Lie is injective. So, we are done with the second proof.

Observe that PreLie $\rightarrow$ Dend is also injective; it has been proved by M. Ronco in [10].

6.6. Splitting of Dend. Since there is an injection ComMag $\rightarrow$ Dend, it is natural to ask the same question as in section 5 , does there exist an $\mathbb{S}$-module $\mathcal{Y}$ such that

$$
\text { Dend }=\mathcal{Y} \circ \text { ComMag } ?
$$

If so, the dimensions of $\mathcal{Y}(n)$ are

$$
1,3,18,168,2130, \ldots \text {. }
$$

More precisely its generating series is $f^{\mathcal{Y}}(t)=\frac{1}{1-3 t-t^{3}}$.

Since, as an $\mathbb{S}$-module, Dend $=$ Dup, and since by [7] we know that Dup = As $\circ M a g$, it would suffice to find $\mathbb{Y}$ such that $M a g=\mathbb{Y} \circ C o m M a g$.

\section{REFERENCES}

[1] N. Bergeron, M. Livernet, The non-symmetric operad pre-Lie is free, Journal of Pure and Applied Algebra 214 (2010), 1165-1172. MR2586994

[2] F. Chapoton, M. Livernet, Pre-Lie algebras and the rooted trees operad. Internat. Math. Res. Notices 2001, no. 8, 395-408. MR.1827084 (2002e:17003)

[3] V. Dotsenko, Freeness theorems for operads via Groebner basis (2010), 15 pp., arXiv: 0907:4958

[4] M. Livernet, A rigidity theorem for pre-Lie algebras, J. Pure Appl. Algebra, 207, 1 (2006), 1-18. MR2244257 (2007g:17001)

[5] J.-L. Loday, Algèbres ayant deux opérations associatives (digèbres). C. R. Acad. Sci. Paris Sér. I Math. 321 (1995), no. 2, 141-146. MR1345436 (96f:16013)

[6] J.-L. Loday, Dialgebras, in "Dialgebras and related operads", Springer Lecture Notes in Math. 1763 (2001), 7-66. MR 1860994 (2002i:17004)

[7] J.-L. Loday, Generalized bialgebras and triples of operads, Astérisque 320 (2008), x+116 pp. MR2504663 (2010f:18007)

[8] J.-L. Loday, B. Vallette, Algebraic operads, in preparation.

[9] M. Markl, Lie elements in pre-Lie algebras, trees and cohomology operations. J. Lie Theory 17 (2007), no. 2, 241-261. MR2325698(2008j:17009)

[10] M. Ronco, Eulerian idempotents and Milnor-Moore theorem for certain non-cocommutative Hopf algebras. J. Algebra 254 (2002), no. 1, 152-172. MR.1927436 (2003f:16064)

[11] M. Markl, S. Shnider, J. Stasheff, Operads in algebra, topology and physics. Mathematical Surveys and Monographs, 96. American Mathematical Society, Providence, RI, 2002. MR1898414 (2003f:18011) 
[12] M. Ronco, Shuffle bialgebras, to appear in Annales Institut Fourier.

[13] K.A. Zhevlakov, A.M. Slinko, I.P. Shestakov, A.I. Shirshov, Rings that are nearly associative. Pure and Applied Mathematics, 104. Academic Press, Inc., 1982. MR668355 (83i:17001)

Department of Mathematics and Statistics, York University, Toronto, Ontario M3J 1P3, CANADA

E-mail address: bergeron@yorku.ca

Institut de Recherche Mathématique Avancée, CNRS et Université de Strasbourg, 7 rue R. Descartes, 67084 Strasbourg Cedex, France

E-mail address: loday@math.unistra.fr 